Open Access

\title{
Robust time-of-arrival source localization employing error covariance of sample mean and sample median in line-of-sight/ non-line-of-sight mixture environments
}

\author{
Chee-Hyun Park ${ }^{1}$ and Joon-Hyuk Chang ${ }^{2 *}$
}

\begin{abstract}
We propose a line-of-sight (LOS)/non-line-of-sight (NLOS) mixture source localization algorithm that utilizes the weighted least squares (WLS) method in LOS/NLOS mixture environments, where the weight matrix is determined in the algebraic form. Unless the contamination ratio exceeds $50 \%$, the asymptotic variance of the sample median can be approximately related to that of the sample mean. Based on this observation, we use the error covariance matrix for the sample mean and median to minimize the weighted squared error (WSE) loss function. The WSE loss function based on the sample median is utilized when statistical testing supports the LOS/NLOS state, while the WSE function using the sample mean is employed when statistical testing indicates that the sensor is in the LOS state. To testify the superiority of the proposed methods, the mean square error (MSE) performances are compared via simulation.
\end{abstract}

Keywords: Adaptive selection, Loss function, Sample mean, Sample median, Statistical testing, Error variance

\section{Introduction}

The aim of the source localization system is to find a geometrical point of intersection using the measurements from each receiver, such as the time difference of arrival (TDOA), time of arrival (TOA), or received signal strength (RSS). Localizing a point source in which passive and stationary sensors are used has been a repeated and popular research issue in the areas of radar, sonar, global positioning system, video conferencing, and telecommunication. Even though location estimation problems have been investigated extensively in the existing literature [1-6], there are still some unresolved problems. One of the key challenges of the localization problem is to estimate the position of the source in dense cluttered nonline-of-sight (NLOS) environments [7, 8]. NLOS scenarios occur when there is an obstruction between transmitters and receivers located in indoor environments and outdoor situations such as urban areas. In general, the research

${ }^{*}$ Correspondence: jchang@hanyang.ac.kr

2 Department of Electronic Engineering, Hanyang University, Seoul 133-791, Republic of Korea

Full list of author information is available at the end of the article fields of localization for the LOS/NLOS mixture problem can be categorized into two parts: (1) the constrained least squares (LS) method using optimization such as the semidefinite relaxation and second-order cone relaxation [9-12] and (2) localization using robust statistics. While localization using the optimization method has comparatively high accuracy, the computational load is higher than that of the analytical solution. Therefore, we concentrate on localization using robust statistics. The existing robust LOS/NLOS mixture position estimators are usually based on the concept of the median, e.g., least median squares (LMedS) [13, 14], $M$-estimator [15, 16], and the HodgesLehmann estimator [17]. The sample mean is an efficient estimator under the normal distribution; thus, it outperforms the accuracy of the sample median in the normal distribution. However, the accuracy of the sample mean is severely degraded when outliers exist in the heavy-tailed distribution, e.g., $t$ distribution or double exponential distribution. On the other hand, the sample median is robust to outliers if the contamination ratio is smaller than $50 \%$ but is inferior to the sample mean when outlier does not exist, i.e., the asymptotic variance of the sample mean approximately amounts to $64 \%$ of variance for the sample 
median when the number of samples is large and the noise distribution follows the normal distribution [18]. Accordingly, the weighted squared error (WSE) loss function based on the sample mean is utilized in the line-of-sight (LOS) condition, where the sensor state is determined by the classical statistical testing $[19,20]$. In contrast, when statistical testing indicates that the sensor in the LOS/NLOS state is valid, the WSE loss function using the sample median is adopted. Then, the source position is determined by minimizing the sum of WSE loss functions based on the sample mean of the sensor expected to be in the LOS condition and sample median of the sensor determined to be in the LOS/NLOS state. The motivation of this paper is as follows. The weighted least squares (WLS) estimator utilizes the weight matrix whose diagonal components amount to the inverse of the error variances of independent noisy measurements. However, the algorithm that utilizes the covariance information of the sample median and mean in the LOS/NLOS mixture state has not yet been reported; that is, the WLS in the LOS/NLOS mixture situation, in which the weight matrix is derived in the algebraic form, has not yet been developed. Thus, we employ the WLS algorithm for the LOS/NLOS mixture state, which is extended from the LOS state, and the diagonal elements of the weight matrix amount to the inverse of asymptotic variances for the sample mean and median. The proposed robust position estimation algorithms differ from the existing two-step WLS algorithm for the LOS conditions, because the proposed methods employ the error variance for the sample median, and this error variance has not been applied to the two-step WLS estimator. The proposed robust localization methods are divided into the iteration method and closed-form algorithm. The Taylor series expansion is utilized in the iteration method, and the two-step WLS algorithm is adopted in the closedform method. The proposed methods exhibit the superior mean square error (MSE) performances when compared to that of existing methods. Moreover, the performance of the proposed closed-form LOS/NLOS mixture localization method using the two-step WLS method is similar to that of the Taylor series-based iteration method, with the advantages of low computational complexity and avoidance of the divergence problem of the solution. When solution diverges, it can reach a solution, which is far from the true solution, or sometimes it fails to produce a solution when the initial value is not appropriately chosen. The LOS/NLOS mixture localization method for multiple sample case has the advantage that all sensors can be utilized compared to the single sample-based LOS/NLOS mixture localization method if the contamination ratio (a measure of how many outliers exist) for the samples in each sensor is lower than the breakdown point (in most cases, when the contamination ratio is less than $10 \%$, the breakdown point of the sample median is $50 \%$ [18]).
The organization of this paper is listed as follows. Section 2 explains the LOS/NLOS mixture source localization problem to be solved in this paper. In Section 3, the details of the existing localization methods using the sample median are addressed. The proposed localization methods using error variances of the sample mean and sample median are addressed in Section 4. The estimation performances of the proposed methods are evaluated via simulation results in Section 5, comparing them with those of the existing algorithms. Finally, the conclusion is presented in Section 6.

\section{Problem formulation}

The main goal of the TOA-based source localization method is to accurately determine the position of a source using multiple circles whose centers are the locations of sensors. In the LOS/NLOS mixture source localization context, the measurement equation is represented as

$$
r_{i, j}=d_{i}+n_{i, j}=\sqrt{\left(x-x_{i}\right)^{2}+\left(y-y_{i}\right)^{2}}+n_{i, j},
$$

where $n_{i, j} \sim(1-\epsilon) N\left(0, \sigma_{1}^{2}\right)+\epsilon N\left(\mu_{2}, \sigma_{2}^{2}\right), i=$ $1,2, \ldots, M, j=1,2, \ldots, P$ with $M$ and $P$ denoting the number of sensors and samples in the $i$ th sensor, respectively [21-23]. Also, $r_{i, j}$ is the measured distance between the source and the $i$ th sensor at the $j$ th sampling and $d_{i}$ is the range (distance) model between the source and $i$ th sensor. The measurement noise $n_{i, j}$ is modeled as a Gaussian mixture distribution in which the LOS noise is distributed according to $N\left(0, \sigma_{1}^{2}\right)$ with a probability $(1-\epsilon)$ and the NLOS noise distributed by $N\left(\mu_{2}, \sigma_{2}^{2}\right)$ with a probability of $\epsilon$. It is assumed that while the statistics of the inlier can be obtained, the mean and variance of the outlier distribution are unknown. Here, $\epsilon(0 \leq \epsilon \leq 1)$ is the contamination ratio (i.e., fraction of contamination) which is a small number (typically smaller than 0.1 ) [21-23]. Also, $\left[\begin{array}{ll}x & y\end{array}\right]^{T}$ is the true source position and $\left[\begin{array}{ll}x_{i} & y_{i}\end{array}\right]^{T}$ is the position of the $i$ th sensor. Note that, throughout this paper, a lowercase boldface letter denotes a vector, an uppercase boldface letter indicates a matrix, and the superscript $T$ signifies the vector/matrix transpose. The purpose of this paper is to determine the source position for which the MSE of the position estimate is minimized.

\section{Review of the existing LOS/NLOS mixture TOA localization methods}

In general, the LS and Gauss-Newton methods have been widely used as localization methods. The two methods are discussed briefly in this section in terms of the formulation of the LOS/NLOS mixture source localization. 


\subsection{LS method using the sample median}

Squaring (1) and rearranging yield the following equation:

$$
\begin{aligned}
x_{i} x+y_{i} y-0.5 R+m_{i, j} & =0.5\left(x_{i}^{2}+y_{i}{ }^{2}-r_{i, j}^{2}\right), \\
i & =1,2, \ldots, M, j=1,2, \ldots, P
\end{aligned}
$$

where $R=x^{2}+y^{2}, m_{i, j}=-d_{i} n_{i, j}-\frac{1}{2} n_{i, j}^{2}$. For convenience, (2) can be simply represented in a matrix form as

$$
\mathbf{A x}+\mathbf{q}_{j}=\mathbf{b}_{j}, j=1, \cdots, P
$$

where $\mathbf{q}_{j}=\left[m_{1, j}, \cdots, m_{M, j}\right]^{T}, \mathbf{x}=\left[\begin{array}{lll}x & y & R\end{array}\right]^{T}$,

$\mathbf{A}=\left(\begin{array}{ccc}x_{1} & y_{1} & -0.5 \\ \vdots & \vdots & \vdots \\ x_{M} & y_{M} & -0.5\end{array}\right)$, and $\mathbf{b}_{j}=\frac{1}{2}\left(\begin{array}{c}x_{1}^{2}+y_{1}^{2}-r_{1, j}^{2} \\ \vdots \\ x_{M}^{2}+y_{M}^{2}-r_{M, j}^{2}\end{array}\right)$

Because the outliers among $r_{i, j}(i=1,2, \cdots, M, j=$ $1,2, \cdots, P$ ) result in severely degraded performance, the median is taken to prevent the adverse effects of outliers. The LS location estimate is then obtained by minimizing the squared error sum as given by

$$
\widehat{\mathbf{x}}=\left(\mathbf{A}^{T} \mathbf{A}\right)^{-1} \mathbf{A}^{T}\left\{\operatorname{med}\left(\mathbf{b}_{1: P}\right)\right\}
$$

where $\operatorname{med}\left(\mathbf{b}_{1: P}\right)$ is $\left[\operatorname{med}\left(b_{1,1: P}\right), \cdots, \operatorname{med}\left(b_{M, 1: P}\right)\right]^{T}$ and med is the abbreviation for the median. Here, (4) differs from the classical LS estimator in which the squared error sum between all samples from each sensor and parametric model is minimized, because (4) minimizes the squared error sum between the median value from each sensor and signal model. The median is the centered value of the ascending ordered sample set. Let us assume the ascending ordered $N$ components of $\left\{a_{(1)}, \cdots, a_{(N)}\right\}$. The median is defined as $a_{((N+1) / 2)}$ if $N$ is odd, or $\left\{a_{(N / 2)}+\right.$ $\left.a_{(N / 2+1)}\right\} / 2$ if $N$ is even. Also, $b_{i, 1: P}$ includes measurements from the first to the $P$ th of the $i$ th sensor. The reason why the median is used to merge the measurements of each sensor is to counteract the effects of outliers.

\subsection{Gauss-Newton method using the sample median}

In the Gauss-Newton method using the sample median, the source position is determined by minimizing the sum of squared error as given below:

$$
\sum_{i=1}^{M}\left(\operatorname{med}\left\{r_{i, 1: P}\right\}-\sqrt{\left(x-x_{i}\right)^{2}+\left(y-y_{i}\right)^{2}}\right)^{2} .
$$

The range is linearized with respect to the reference point $\mathbf{x}_{0}$ using the Taylor series method as follows:

$$
\mathbf{d}=\mathbf{f}(\mathbf{x}) \simeq \mathbf{f}\left(\mathbf{x}_{0}\right)+\mathbf{G}\left(\mathbf{x}-\mathbf{x}_{0}\right)
$$

where $\mathbf{d}=\left[d_{1} \cdots d_{M}\right]^{T}, \mathbf{f}(\mathbf{x})=\left[f_{1}(x) \cdots f_{M}(x)\right]^{T}, f_{i}(\mathbf{x})=$ $\sqrt{\left(x-x_{i}\right)^{2}+\left(y-y_{i}\right)^{2}}$, and

$$
\mathbf{G}=\left(\begin{array}{cc}
\left.\frac{\partial f_{1}}{\partial x}\right|_{\mathbf{x}=\mathbf{x}_{0}} & \left.\frac{\partial f_{1}}{\partial y}\right|_{\mathbf{x}=\mathbf{x}_{0}} \\
\vdots & \vdots \\
\left.\frac{\partial f_{M}}{\partial x}\right|_{\mathbf{x}=\mathbf{x}_{0}} & \left.\frac{\partial f_{M}}{\partial y}\right|_{\mathbf{x}=\mathbf{x}_{0}}
\end{array}\right)
$$

Each row of matrix $\mathbf{G}$ is the gradient vector of one of the components of $\mathbf{f}(\mathbf{x})$. The vector $\mathbf{x}_{\mathrm{o}}$ could be an estimate of $\mathbf{x}$ determined from the previous iteration. The source position is then obtained using the same procedure in [1] as follows:

$$
\mathbf{x}^{(k+1)}=\mathbf{x}^{(k)}+\left(\mathbf{G}^{(k)^{T}} \mathbf{G}^{(k)}\right)^{-1} \mathbf{G}^{(k)^{T}}\left(\operatorname{med}\left(\mathbf{r}_{1: P}\right)-\mathbf{f}\left(\mathbf{x}^{(k)}\right)\right)
$$

where $\mathbf{x}^{(k)}$ is the estimate of $\mathbf{x}$ in the $k$ th iteration (the superscript $(k)$ denotes the iteration number) and $\operatorname{med}\left(\mathbf{r}_{1: P}\right)=\left[\operatorname{med}\left(r_{1,1: P}\right), \cdots, \operatorname{med}\left(r_{M, 1: P}\right)\right]^{T}$.

\section{Proposed adaptive LOS/NLOS mixture TOA source localization method using the WSE loss function}

In this paper, the LOS/NLOS mixture state is divided into the LOS and LOS/NLOS states. The LOS state denotes the case where the contamination ratio is zero $(\varepsilon=0)$ and the LOS/NLOS state is the condition in which $0<\varepsilon \leq 1$. The WSE based on the sample median is robust to outliers as the loss function when the sensor is in the LOS/NLOS state but is less efficient than the WSE loss function using the sample mean when the sensor is in the LOS condition. Hence, it is adequate to adaptively select the loss function by investigating whether the corresponding sensor is in the LOS or LOS/NLOS condition. To determine whether outliers exist among measurements of the sensor, some testing rules to be explained later are used.

\subsection{Adaptive LOS/NLOS mixture localization using the WSE loss function}

At first, the proposed adaptive LOS/NLOS mixture localization method uses the WSE loss function based on the sample mean when the sensor is judged by the LOS sensor and the WSE loss function using the sample median 
if the sensor is predicted to be the LOS/NLOS sensor. Hereafter, this method is referred to as the ANLOS method. When the sensor is in the LOS condition and the noise follows a normal distribution, it is known that the asymptotic relative efficiency represented as $\frac{\operatorname{Var}(\bar{x})}{\operatorname{Var}(\bar{x})}(\bar{x}$ and $\tilde{x}$ are respectively the sample mean and sample median and Var is the abbreviation for the variance) is $2 / \pi \simeq 0.64$ when the number of samples is large [18]. That is, the WSE loss function based on the sample mean is more efficient than that using the sample median when no outlier exists. Thus, the proposed adaptive method utilizes the WSE based on the sample mean as the loss function if the testing result that the sensor is in the LOS condition is supported. To select the proper loss function according to the state of each sensor, discrimination between the LOS and LOS/NLOS sensor must be performed. When no outlier exists, the difference between the sample mean and range measurements of the $i$ th sensor would not be inflated by outliers; thus, the difference between the sample mean and range measurements of the $i$ th sensor when the outlier does not exist would be smaller than that in the case that the outlier exists. Hence, the following statistical testing is reasonable to determine whether an outlier exists or not

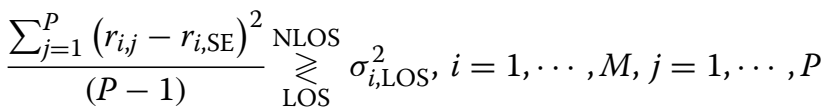

where $r_{i, \mathrm{SE}}=\frac{1}{P} \sum_{j=1}^{P} r_{i, j}[19]$. In practice, $\sigma_{i, \mathrm{LOS}}^{2}$ can be estimated by observing the energy bins in an absence of the transmitted signal [24]. When the left-hand side of (8) is smaller than $\sigma_{i, \mathrm{LOS}}^{2}$ (the variance of the single sample of the $i$ th LOS sensor), the following WSE loss function based on the sample mean is used since the judgment that the $i$ th sensor is in the LOS condition is supported

$$
\frac{1}{\sigma_{i, \mathrm{SE}}^{2}}\left(r_{i, \mathrm{SE}}-f_{i}\left(\mathbf{x}_{\mathrm{o}}\right)-\mathbf{g}_{i}^{T} \mathbf{x}+\mathbf{g}_{i}^{T} \mathbf{x}_{\mathrm{o}}\right)^{2}
$$

where $\mathbf{g}_{i}^{T}$ denotes the $i$ th row of $\mathbf{G}$, represented as $\left[\left.\left.\frac{\partial f_{i}}{\partial x}\right|_{\mathbf{x}=\mathbf{x}_{0}} \frac{\partial f_{i}}{\partial y}\right|_{x=\mathbf{x}_{0}}\right], \sigma_{i, \mathrm{SE}}^{2}$ is the variance of the sample mean of the measurements in the $i$ th LOS sensor and is found by $\frac{\sigma_{i, \mathrm{LOS}}^{2}}{P}$. This WSE loss function is extended to the multiple sensor case as follows:

$$
\sum_{i \in \Lambda} \frac{1}{\sigma_{i, \mathrm{SE}}^{2}}\left(r_{i, \mathrm{SE}}-f_{i}\left(\mathbf{x}_{\mathrm{o}}\right)-\mathbf{g}_{i}^{T} \mathbf{x}+\mathbf{g}_{i}^{T} \mathbf{x}_{\mathrm{o}}\right)^{2}
$$

where $\Lambda$ denotes the sensor index set that belongs to the LOS sensors. On the contrary, when the left-hand side of
(8) is larger than $\sigma_{i, \mathrm{LOS}}^{2}$, the WSE using the sample median is utilized as the loss function because the $q$ th sensor is assumed to be in the LOS/NLOS condition and can be extended to the multiple sensor case as given below:

$$
\sum_{q \in \Lambda^{c}} \frac{1}{\sigma_{q, \mathrm{SA}}^{2}}\left(\operatorname{med}\left(r_{q, 1: P}\right)-f_{q}\left(\mathbf{x}_{\mathrm{o}}\right)-\mathbf{g}_{q}^{T} \mathbf{x}+\mathbf{g}_{q}^{T} \mathbf{x}_{\mathrm{o}}\right)^{2}
$$

where $\Lambda^{c}$ is the sensor index set belonging to the LOS/NLOS sensors and $\sigma_{q, \mathrm{SA}}^{2}$ is the variance of the sample median for the measurements in the $q$ th LOS/NLOS sensor. When the contamination ratio does not exceed $50 \%, \sigma_{q, \mathrm{SA}}^{2}$ can be approximated by $\frac{\pi}{2} \frac{\sigma_{q, \mathrm{LOS}}^{2}}{P}\left(\sigma_{q, \mathrm{LOS}}^{2}\right.$ is the variance of the single LOS sample of the $q$ th LOS/NLOS sensor) [25]. Then, the source location parameter is determined by minimizing the sum of (10) and (11) as follows:

$$
\begin{aligned}
\min & \sum_{i \in \Lambda} \frac{1}{\sigma_{i, \mathrm{SE}}^{2}}\left(r_{i, \mathrm{SE}}-f_{i}\left(\mathbf{x}_{\mathrm{o}}\right)-\mathbf{g}_{i}^{T} \mathbf{x}+\mathbf{g}_{i}^{T} \mathbf{x}_{\mathrm{o}}\right)^{2} \\
& +\sum_{q \in \Lambda^{c}} \frac{1}{\sigma_{q, \mathrm{SA}}^{2}}\left(\operatorname{med}\left(r_{q, 1: P}\right)-f_{q}\left(\mathbf{x}_{\mathrm{o}}\right)-\mathbf{g}_{q}^{T} \mathbf{x}+\mathbf{g}_{q}^{T} \mathbf{x}_{\mathrm{o}}\right)^{2} .
\end{aligned}
$$

The location parameter that satisfies (12) is determined as follows:

$$
\begin{aligned}
\mathbf{x}^{(k+1)}= & \mathbf{x}^{(k)}+\left(\sum_{i \in \Lambda} \frac{1}{\sigma_{i, \mathrm{SE}}^{2}}\left\{\mathbf{g}_{i}^{(k)} \mathbf{g}_{i}^{(k)^{T}}\right\}\right. \\
& \left.+\sum_{q \in \Lambda^{c}} \frac{1}{\sigma_{q, \mathrm{SA}}^{2}}\left\{\mathbf{g}_{q}^{(k)} \mathbf{g}_{q}^{(k)}\right\}\right)^{-1} \\
& \times\left(\sum_{i \in \Lambda} \mathbf{g}_{i}^{(k)} \frac{1}{\sigma_{i, \mathrm{SE}}^{2}}\left(r_{i, \mathrm{SE}}-f_{i}\left(\mathbf{x}^{(k)}\right)\right)\right. \\
& \left.+\sum_{q \in \Lambda^{c}} \mathbf{g}_{q}^{(k)} \frac{1}{\sigma_{q, \mathrm{SA}}^{2}}\left(\operatorname{med}\left(r_{q, 1: P}\right)-f_{q}\left(\mathbf{x}^{(k)}\right)\right)\right) .
\end{aligned}
$$

\subsection{Adaptive LOS/NLOS mixture two-step WLS localization using the WSE loss function}

While the previous proposed method is the iterationbased method, the closed-form location estimator is developed in this section. This proposed closed-form method is referred to as the ATWLS method. The WLS estimator in LOS/NLOS mixture environments, where the 
weight is derived by mathematical analysis, has not yet been investigated. In (3), this algorithm substitutes the sample mean of $r_{i, 1: P}$ into $r_{i}$ if the testing result that the sensor belongs to the LOS sensor is valid or replaces the sample median of $r_{i, 1: P}$ inside $r_{i}$ when the sensor is determined to be the LOS/NLOS sensor as follows:

$$
\begin{aligned}
& \widehat{b}_{i}=0.5\left(x_{i}{ }^{2}+y_{i}{ }^{2}-\left(r_{i, \mathrm{SE}}\right)^{2}\right), i \in \Lambda \\
& \widehat{b}_{q}=0.5\left(x_{q}{ }^{2}+y_{q}{ }^{2}-\left(r_{q, \mathrm{SA}}\right)^{2}\right), q \in \Lambda^{c}
\end{aligned}
$$

where $r_{q, \mathrm{SA}}=\operatorname{med}\left(r_{q, 1: P}\right)$. The covariance matrix (weight matrix) of $\widehat{\mathbf{b}}=\left[\widehat{b}_{1}, \cdots, \widehat{b}_{M}\right]^{T}$ should be determined to derive the WLS estimator. Since $\frac{1}{P} \sum_{j=1}^{P} r_{i, j}=d_{i}+$ $\frac{1}{P} \sum_{j=1}^{P} n_{i, j}$, the error statistics of $\widehat{b}_{i}$ (defined as $\Delta \widehat{b}_{i}$ ) is found from (14) as follows:

$$
\begin{aligned}
\Delta \widehat{b}_{i} & =-\frac{1}{2}\left\{\frac{2 d_{i}}{P} \sum_{j=1}^{P} n_{i, j}+\left(\frac{1}{P} \sum_{j=1}^{P} n_{i, j}\right)^{2}\right\}, \\
& \simeq-\frac{d_{i}}{P} \sum_{j=1}^{P} n_{i, j}, \\
& \simeq-\frac{r_{i, \mathrm{SE}}}{P} \sum_{j=1}^{P} n_{i, j}, i \in \Lambda .
\end{aligned}
$$

In the derivation from the first to the second equation of (15), the LOS noise was assumed to be sufficiently small, and from the second to the third equation, $r_{i}$,SE was substituted into $d_{i}$ because $d_{i}$ is the unknown value. Then, $\sigma_{\widehat{b}_{i}}^{2}$ is found as $\frac{r_{i, \mathrm{SE}}^{2} \sigma_{i}^{2}}{P}$ for the sensors determined to be LOS sensor. Meanwhile, when $r_{q, \mathrm{SA}}=\operatorname{med}\left(r_{q, 1: P}\right)=$ $d_{q}+\operatorname{med}\left(n_{q, 1: P}\right)$, the noise of $\widehat{b}_{q}\left(\Delta \widehat{b}_{q}\right)$ for the sensors predicted to be the LOS/NLOS sensor is obtained from (14) as follows:

$$
\begin{aligned}
\Delta \widehat{b}_{q} & =-\frac{1}{2}\left\{2 d_{q} \operatorname{med}\left(n_{q, 1: P}\right)+\left(\operatorname{med}\left(n_{q, 1: P}\right)\right)^{2}\right\} \\
& \simeq-r_{q, \mathrm{SA}} \cdot \operatorname{med}\left(n_{q, 1: P}\right), q \in \Lambda^{c} .
\end{aligned}
$$

In (16), $r_{q, \mathrm{SA}}$ was substituted instead of $d_{q}$ and a sufficiently small LOS noise condition was assumed. As in the previously proposed methods, $\sigma_{\widehat{b}_{q}}^{2}$ can be approximated as $\frac{\pi}{2} \frac{r_{q, S A}^{2} \sigma_{q}^{2}}{P}$ when the contamination ratio does not exceed $50 \%$. The first-step WLS estimator is then obtained by minimizing the sum of WSE loss functions based on the sample mean and sample median as follows:

$$
\min \sum_{i \in \Lambda} \frac{1}{\sigma_{b_{i}}^{2}}\left(\widehat{b}_{i}-\mathbf{a}_{i}^{T} \mathbf{x}\right)^{2}+\sum_{q \in \Lambda^{c}} \frac{1}{\sigma_{b_{q}}^{2}}\left(\widehat{b}_{q}-\mathbf{a}_{q}^{T} \mathbf{x}\right)^{2}
$$

where $\mathbf{a}_{i}^{T}$ is the $i$ th row of $\mathbf{A}$. Then, the first-step WLS estimator is obtained as shown below:

$$
\widehat{\mathbf{x}}_{1}=\left(\mathbf{A}^{T} \mathbf{C}_{\widehat{\mathbf{b}}}^{-1} \mathbf{A}\right)^{-1} \mathbf{A}^{T} \mathbf{C}_{\widehat{\mathbf{b}}}^{-1} \widehat{\mathbf{b}},
$$

where

$$
\left[\mathbf{C}_{\widehat{\mathbf{b}}}\right]_{i, j}= \begin{cases}\frac{r_{i, \mathrm{SE}}^{2} \sigma_{i}^{2}}{P}, & \text { if } i=j \text { and LOS sensor, } \\ \frac{\pi}{2} \frac{r_{i, \mathrm{SA}}^{2} \sigma_{i}^{2}}{P}, & \text { if } i=j \text { and LOS/NLOS sensor, } \\ 0, & \text { if } i \neq j .\end{cases}
$$

The first-step WLS estimate (18) can be further improved using the two-step WLS estimator [2,3], which is represented as follows:

$$
\widehat{\mathbf{x}}_{2}=\left(\mathbf{H}^{T} \mathbf{C}_{\widehat{\mathbf{h}}}^{-1} \mathbf{H}\right)^{-1} \mathbf{H}^{T} \mathbf{C}_{\widehat{\mathbf{h}}}^{-1} \widehat{\mathbf{h}}
$$

where

$$
\begin{aligned}
\widehat{\mathbf{h}}= & {\left[\left[\widehat{\mathbf{x}}_{1}\right]_{1}^{2}\left[\widehat{\mathbf{x}}_{1}\right]_{2}^{2}\left[\widehat{\mathbf{x}}_{1}\right]_{3}\right]^{T}, \mathbf{C}_{\widehat{\mathbf{h}}}=\operatorname{diag}\left[\begin{array}{lll}
2 x & 2 y & 1
\end{array}\right] } \\
& \times\left(\mathbf{A}^{T} \mathbf{C}_{\widehat{\mathbf{b}}}^{-1} \mathbf{A}\right)^{-1} \operatorname{diag}\left[\begin{array}{lll}
2 x & 2 y & 1
\end{array}\right], \text { and } \\
\mathbf{H}= & \left(\begin{array}{ll}
1 & 0 \\
0 & 1 \\
1 & 1
\end{array}\right)
\end{aligned}
$$

$[\cdot]_{k}$ means the $k$ th element of $[\cdot]$, and $x, y$ are substituted as $\left[\mathbf{x}_{1}\right]_{1},\left[\mathbf{x}_{1}\right]_{2}$ in the computation of $\mathbf{C}_{\widehat{\mathbf{h}}}$. Namely, the localization performance can be improved using the second-step solution since it adopts the error distribution of the first-step estimate. The final closed-form two-step WLS source location estimate is found as follows:

$$
\widehat{\mathbf{x}}_{f}=\left[\operatorname{sgn}\left(\left[\widehat{\mathbf{x}}_{1}\right]_{1}\right) \sqrt{\left[\widehat{\mathbf{x}}_{2}\right]_{1}} \operatorname{sgn}\left(\left[\widehat{\mathbf{x}}_{1}\right]_{2}\right) \sqrt{\left[\widehat{\mathbf{x}}_{2}\right]_{2}}\right]^{T}
$$

where $\operatorname{sgn}(\cdot)$ denotes the sign function.

\subsection{The ATWLS algorithm in the presence of sensor position error}

Sensor position error may exist in practical environments and leads to degraded localization performance [26, 27]. In this section, we present a localization algorithm that can be applied to environments with sensor position errors. The measurement equation when the sensor position errors exist is represented as follows [26]:

$$
r_{i, j}=\sqrt{\left(x-x_{i}^{\mathrm{o}}\right)^{2}+\left(y-y_{i}^{\mathrm{o}}\right)^{2}}+n_{i, j}
$$


where $x_{i}^{\mathrm{o}}$ and $y_{i}^{\mathrm{o}}$ are the true coordinates of the $i$ th sensor and the erroneous sensor positions are represented as $x_{i}=x_{i}^{\mathrm{o}}+\Delta x_{i}, y_{i}=y_{i}^{\mathrm{o}}+\Delta y_{i},\left[\Delta x_{i}, \Delta y_{i}\right]^{T}$ denote the sensor position error of the $i$ th sensor. Squaring (21) and substituting $x_{i}^{\mathrm{o}}=x_{i}-\Delta x_{i}, y_{i}^{\mathrm{o}}=y_{i}-\Delta y_{i}$ yield the following [26]:

$$
\begin{gathered}
\frac{x_{i}^{2}+y_{i}^{2}-r_{i, j}^{2}}{2}-\left[\begin{array}{lll}
x_{i} & y_{i}-0.5
\end{array}\right]\left[\begin{array}{l}
x \\
y \\
R
\end{array}\right] \\
=\left(x_{i}-x\right) \Delta x_{i}+\left(y_{i}-y\right) \Delta y_{i}-d_{i} n_{i, j} \\
(i=1, \cdots, M, j=1, \cdots, P) .
\end{gathered}
$$

The second-order noise terms were neglected in the derivation of (22) because they are assumed to be small values; (22) can then be represented in a matrix form as

$$
\mathbf{Q x}+\mathbf{e}_{j}=\mathbf{t}_{j}, j=1, \cdots, P
$$

where $\mathbf{e}_{j}=\left[e_{1, j}, \cdots, e_{M, j}\right]^{T}, e_{i, j}=\left(x_{i}-x\right) \Delta x_{i}+\left(y_{i}-y\right) \Delta y_{i}-$ $d_{i} n_{i, j}, \mathbf{x}=\left[\begin{array}{lll}x & y & R\end{array}\right]^{T}$,

$\mathbf{Q}=\left(\begin{array}{ccc}x_{1} & y_{1} & -0.5 \\ \vdots & \vdots & \vdots \\ x_{M} & y_{M} & -0.5\end{array}\right)$, and $\mathbf{t}_{j}=\frac{1}{2}\left(\begin{array}{c}x_{1}^{2}+y_{1}^{2}-r_{1, j}^{2} \\ \vdots \\ x_{M}^{2}+y_{M}^{2}-r_{M, j}^{2}\end{array}\right)$

The localization algorithm performs the testing described in (8) and determines the position using the two-step WLS estimator as in Section 4.2. The error covariance matrix in the first-step WLS estimator is determined as follows:

$$
\left[\mathbf{C}_{\hat{\mathbf{t}}}\right]_{i, j}= \begin{cases}\frac{r_{i, \mathrm{SE}}^{2} \sigma_{i}^{2}}{P}+r_{i, \mathrm{SE}}^{2} \sigma_{\Delta s}^{2}, & \text { if } \mathrm{i}=\mathrm{j} \text { and LOS sensor, } \\ \frac{\pi}{2} \frac{r_{i, \mathrm{SA}}^{2} \sigma_{i}^{2}}{P}+r_{i, \mathrm{SA}}^{2} \sigma_{\Delta s}^{2}, & \text { if } i=j \text { and LOS/NLOS sensor, } \\ 0, & \text { if } i \neq j .\end{cases}
$$

where $\widehat{\mathbf{t}}=\left[\widehat{t}_{1}, \cdots, \widehat{t}_{M}\right]^{T}, \widehat{t}_{i}=0.5\left(x_{i}{ }^{2}+y_{i}{ }^{2}-\left(r_{i, \mathrm{SE}}\right)^{2}\right)$, if $i \in \Lambda, \widehat{t}_{q}=0.5\left(x_{q}{ }^{2}+y_{q}{ }^{2}-\left(r_{q, \mathrm{SA}}\right)^{2}\right)$, if $q \in \Lambda^{c}$ and $\sigma_{\Delta s}^{2}$ is the variance of the sensor position error. In (24), we substitute the sample mean and median value into $d_{i}(i=1, \cdots, M)$ because the true distance values are unknown. It is assumed that $\sigma_{\Delta x_{i}}^{2}=\sigma_{\Delta y_{i}}^{2}=\sigma_{\Delta s}^{2}$ and $\Delta x_{i}, \Delta y_{i}$ and $n_{i, j}$ are uncorrelated in the derivation of (24), where $\sigma_{\Delta x_{i}}^{2}, \sigma_{\Delta y_{i}}^{2}$ are the variances of $x, y$ position error of the $i$ th sensor. The algorithms used in this paper are summarized in Table 1 as follows.
ATWLS

LMedS

Table 1 Summary of the localization algorithms

$$
\begin{array}{ll}
\hline \text { Algorithm } \quad \text { Solution } & \\
\hline \text { LS (median) } \quad\left(\mathbf{A}^{\top} \mathbf{A}\right)^{-1} \mathbf{A}^{\top}\left\{\operatorname{med}\left(\mathbf{b}_{1: P}\right)\right\} & \\
\text { ANLOS } \quad \mathbf{x}^{(k+1)}= & \mathbf{x}^{(k)}+\left(\sum_{i \in \Lambda} \frac{1}{\sigma_{i, S E}^{2}}\left\{\mathbf{g}_{i}^{(k)} \mathbf{g}_{i}^{(k)}\right\}_{1}^{T}\right. \\
& \left.+\sum_{q \in \Lambda^{c}} \frac{1}{\sigma_{q, S \mathrm{~A}}^{2}}\left\{\mathbf{g}_{q}^{(k)} \mathbf{g}_{q}^{(k)^{T}}\right\}\right)^{-1} \\
& \times\left(\sum_{i \in \Lambda} \mathbf{g}_{i}^{(k)} \frac{1}{\sigma_{i, S E}^{2}}\left(r_{i, S E}-f_{i}\left(\mathbf{x}^{(k)}\right)\right)\right. \\
& \left.+\sum_{q \in \Lambda^{c}} \mathbf{g}_{q}^{(k)} \frac{1}{\sigma_{q, S \mathrm{~S}}^{2}}\left(\operatorname{med}\left(\mathbf{r}_{q, 1: P}\right)-f_{q}\left(\mathbf{x}^{(k)}\right)\right)\right)
\end{array}
$$

ATLS

$\left(\mathbf{A}^{T} \mathbf{A}\right)^{-1} \mathbf{A}^{\top} \widehat{\mathbf{b}}$

Gauss-Newton

$\mathbf{x}^{(k+1)}=\mathbf{x}^{(k)}+\left(\sum_{q=1}^{M} \frac{1}{\sigma_{q, S A}^{2}}\left\{\mathbf{g}_{q}^{(k)} \mathbf{g}_{q}^{(k)}\right\}\right)^{-1}$

$\times\left(\sum_{q=1}^{M} \mathbf{g}_{q}^{(k)} \frac{1}{\sigma_{q, S A}^{2}}\left(\operatorname{med}\left(\mathbf{r}_{q, 1: P}\right)-f_{q}\left(\mathbf{x}^{(k)}\right)\right)\right)$

M-estimator

$\min _{\mathbf{x}}\left\{\sum_{i=1}^{M} \rho\left(r_{i}(\mathbf{x})\right)\right\}$

$\rho(t)=\frac{1}{2} t^{2}$ if $|t| \leq \gamma, \rho(t)=\gamma|t|-\frac{1}{2} \gamma^{2}$ elseif $|t|>\gamma$,

$r_{i}(\mathbf{x})=\left[\operatorname{med}\left(\mathbf{b}_{1: P}\right)\right]_{i}-[\mathbf{A x}]_{i},[\cdot]_{i}$ denotes the ith component of $[\cdot]$,

$\gamma$ is the tuning constant $(\gamma>0)$.

$\left(\mathbf{A}^{T} \mathbf{C}_{\widehat{\mathbf{b}}}^{-1} \mathbf{A}\right)^{-1} \mathbf{A}^{T} \mathbf{C}_{\widehat{\mathbf{b}}}^{-1} \widehat{\mathbf{b}}$

(1) Calculate the $m$ subsets of three measurements.

(2) For each subset $\mathrm{S}$, compute a location by trilateration in closed-form $L S$ solution $\left(\left[\widehat{x}_{L S}, \widehat{y}_{L S}\right]^{T}\right)$.

(3) For each solution, the residues $\mathbf{R}_{s}$ are

obtained as $\mathbf{R}_{5}=\left[\left(r_{1}-\widehat{r}_{1}\right)^{2},\left(r_{2}-\widehat{r}_{2}\right)^{2}, \ldots\right.$,

$\left.\left(r_{M}-\widehat{r}_{M}\right)^{2}\right]^{T}$

where $\widehat{r}_{i}=\sqrt{\left(\widehat{x}_{L S}-x_{i}\right)^{2}+\left(\hat{y}_{L S}-y_{i}\right)^{2}}$

$(i=1, \cdots, M)$

and the median of the residues is obtained.

(4) Find the minimum median of the residues.

\section{Simulation results}

In this section, the MSE performances of the proposed LOS/NLOS mixture source localization methods are compared with those of the $M$-estimator [16] and LMedS estimator [28]. In this simulation settings, the source was assumed to be located within a $400-\mathrm{m}^{2}$ region to determine the performance over the entire area. Note that the number of sensors used in this experiment was seven. Next, ten different source locations were generated with a uniform distribution and sensors fixed, as shown in Fig. 1. Two hundred Monte-Carlo simulations were performed for each given standard deviation of the NLOS noise. The standard deviation of the LOS noise of all of the sensors 


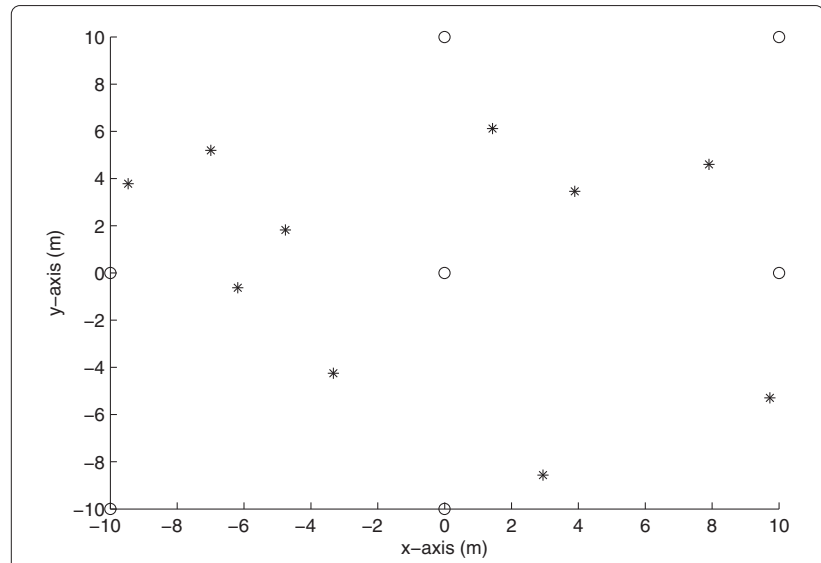

Fig. 1 Sources utilized in the simulation when the number of sensors is seven (white circle as sensors, asterisks as sources)

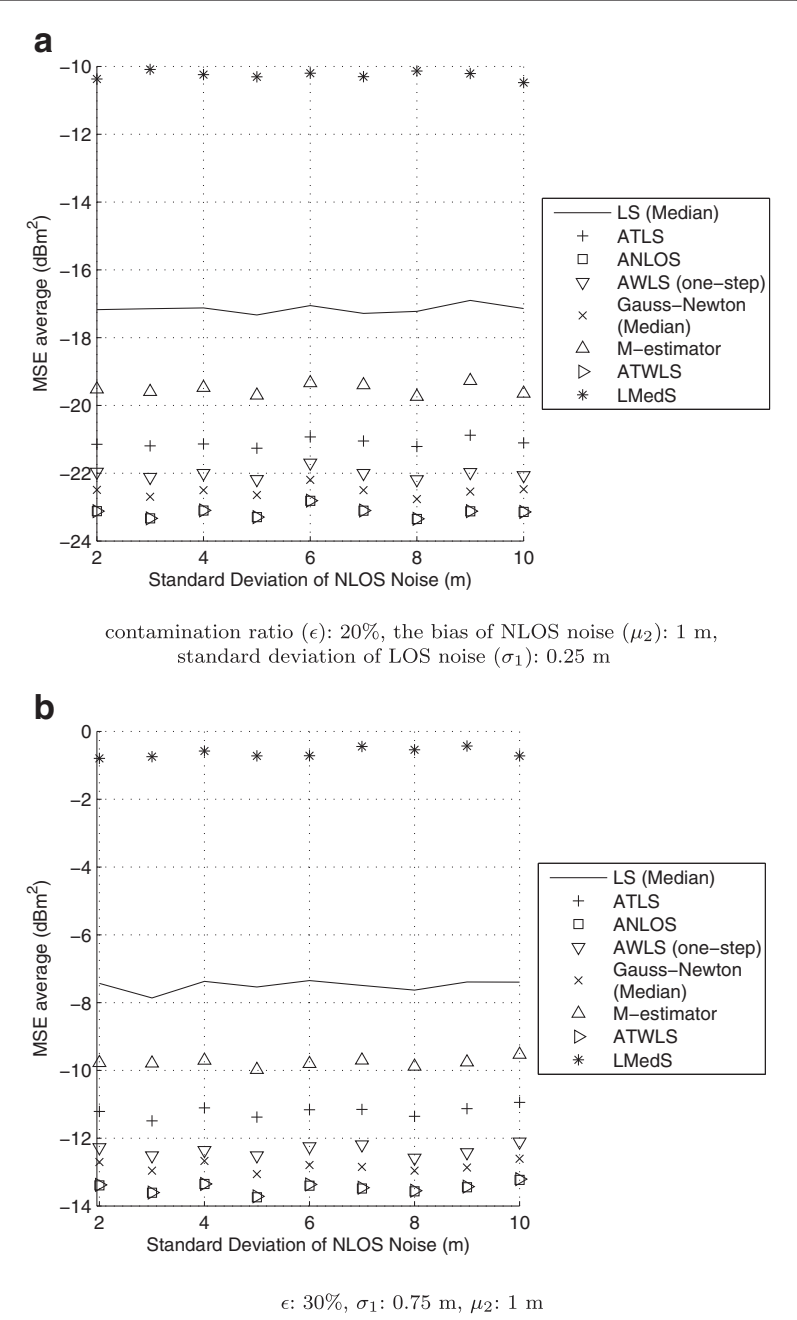

Fig. 2 Comparison of MSE averages of the proposed estimators with that of existing methods when sensor 3 is the LOS/NLOS sensor and the remaining sensors are the LOS sensors was assumed to be identical. In addition, the single and omni-directional source was assumed to be in the stationary state. The MSE average was calculated as follows:

MSE average $=$

$$
\frac{\sum_{i=1}^{10} \sum_{k=1}^{200}\left[\left(\widehat{x}^{k}(i)-x(i)\right)^{2}+\left(\widehat{y}^{k}(i)-y(i)\right)^{2}\right]}{10 \times 200}
$$

where $\widehat{x}^{k}(i), \hat{y}^{k}(i)$ is the estimated position of the source in the $i$ th position set and $k$ th iteration and $x(i)$ and $y(i)$ indicate the $i$ th true position of the source.

Figure 1 illustrates a deployment of sensors, in which the radius of the sensor network was set to $10 \mathrm{~m}$. The localization accuracy as a function of the standard deviation of the NLOS noise is shown in Fig. 2 when the radius of the sensor network was $10 \mathrm{~m}$. In Fig. 2a, the contamination ratio $(\epsilon)$ was $20 \%$, the standard deviation of the

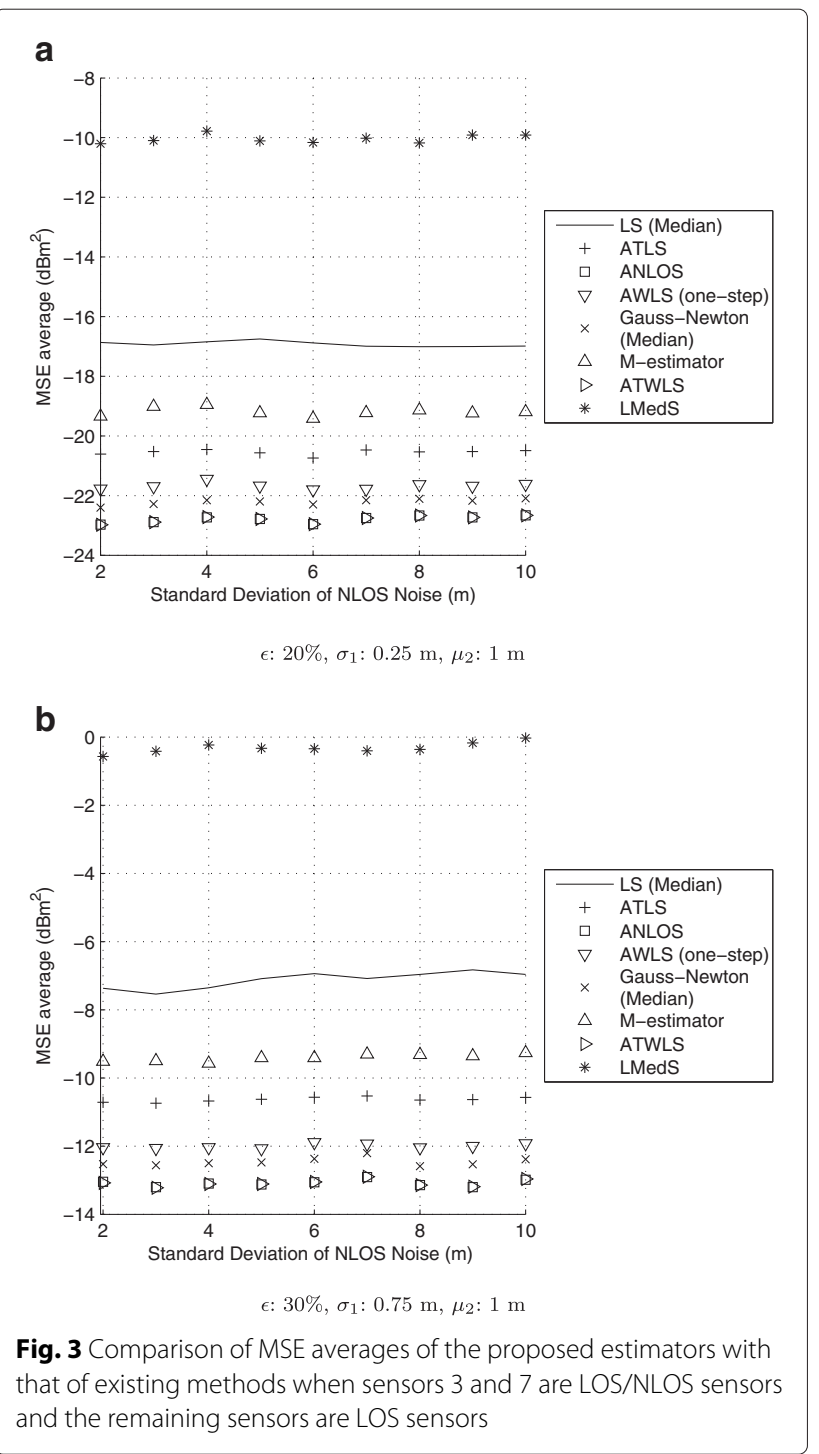



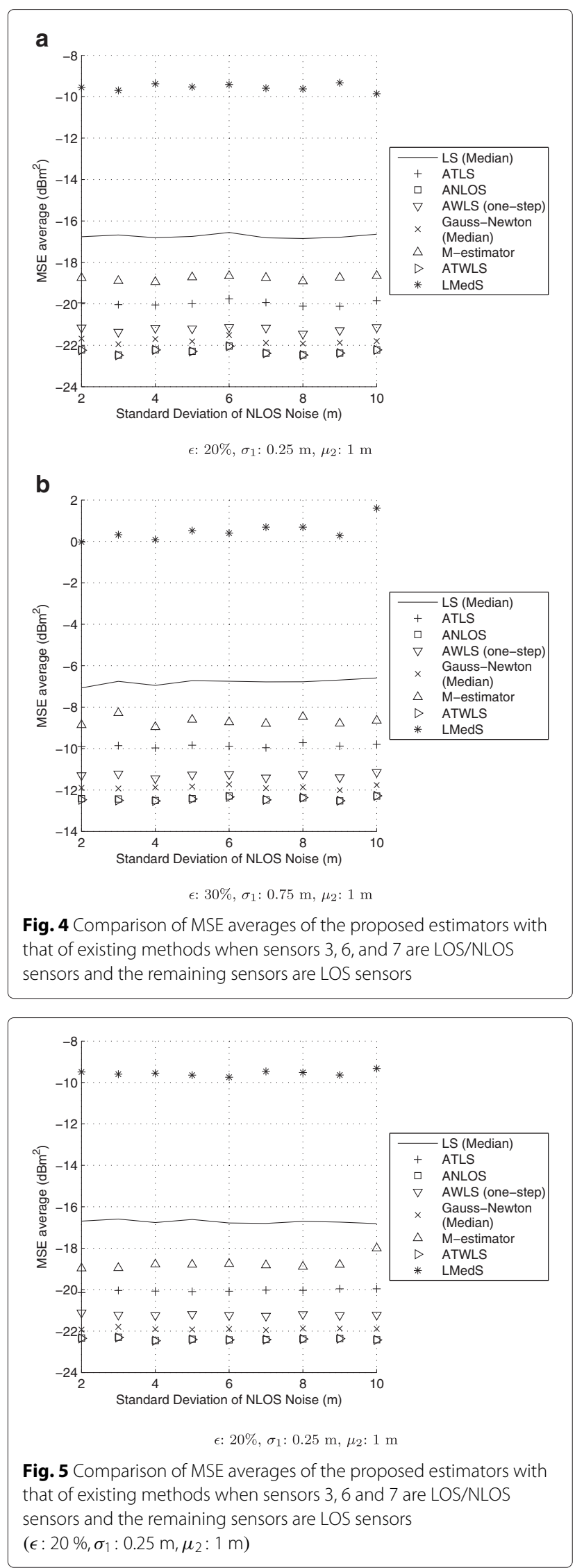

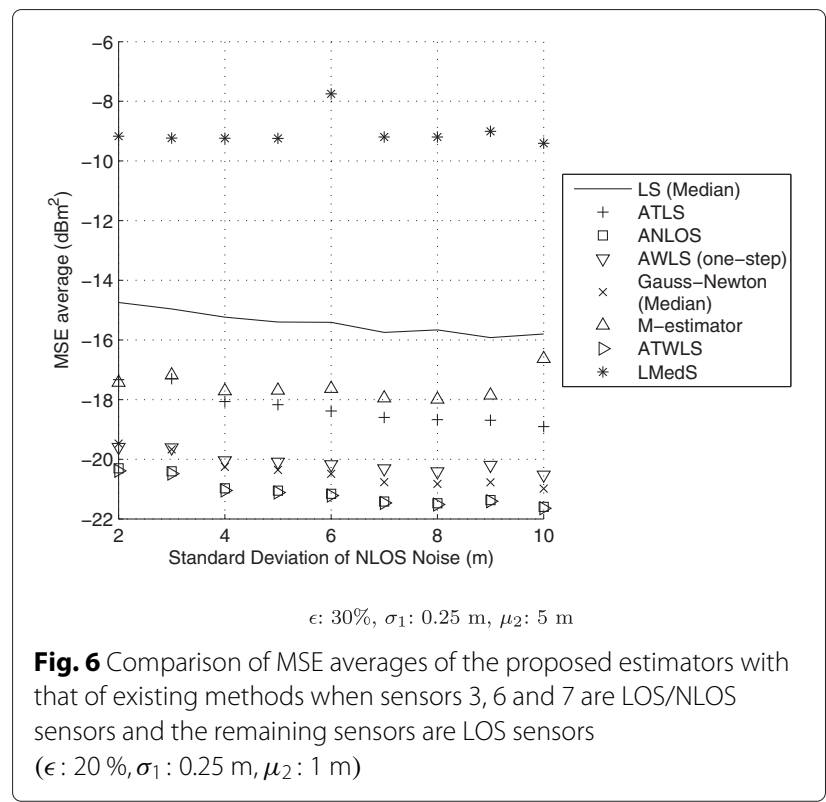

LOS noise $\left(\sigma_{1}\right)$ was $0.25 \mathrm{~m}$, the bias of the NLOS noise $\left(\mu_{2}\right)$ was $1 \mathrm{~m}$, sensor 3 was the LOS/NLOS sensor, and the remaining sensors were LOS sensors at which the number of measurements in each sensor was ten. It is clear that the MSE averages of the proposed methods, the ANLOS and ATWLS algorithms, are lower than those of the nonadaptive LS, adaptive two-step LS (ATLS), adaptive onestep WLS (AWLS (one-step)), Gauss-Newton methods using the sample median and $M$-estimator. In Fig. 2b, the contamination ratio was $30 \%$, the standard deviation of the LOS noise is $0.75 \mathrm{~m}$, and the remaining conditions are the same as those in Fig. 2a. Figure 2b shows that the MSE average performances of the proposed methods are much superior to those of the other methods. The weight matrix

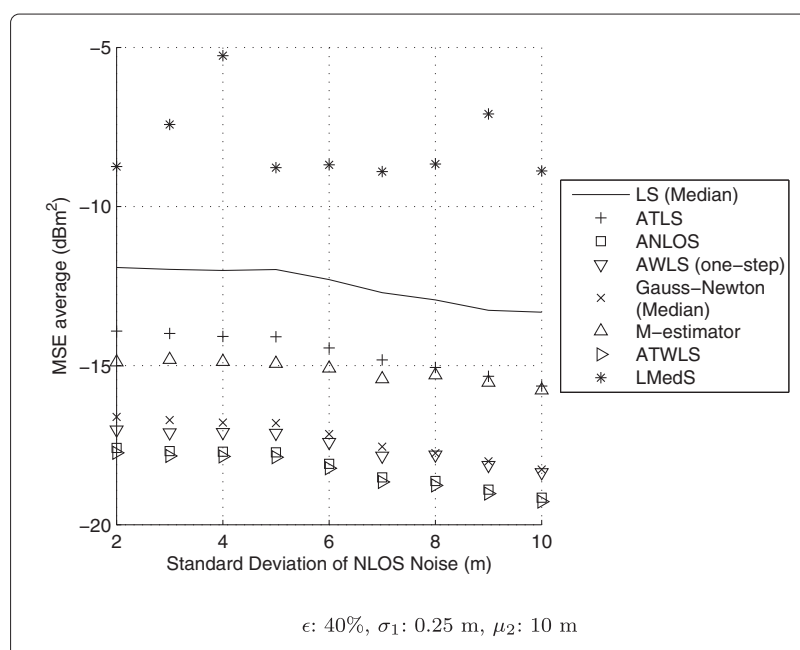

Fig. 7 Comparison of MSE averages of the proposed estimators with that of existing methods when sensors 3, 6 and 7 are LOS/NLOS sensors and the remaining sensors are LOS sensors $\left(\epsilon: 20 \%, \sigma_{1}: 0.25 \mathrm{~m}, \mu_{2}: 1 \mathrm{~m}\right)$ 


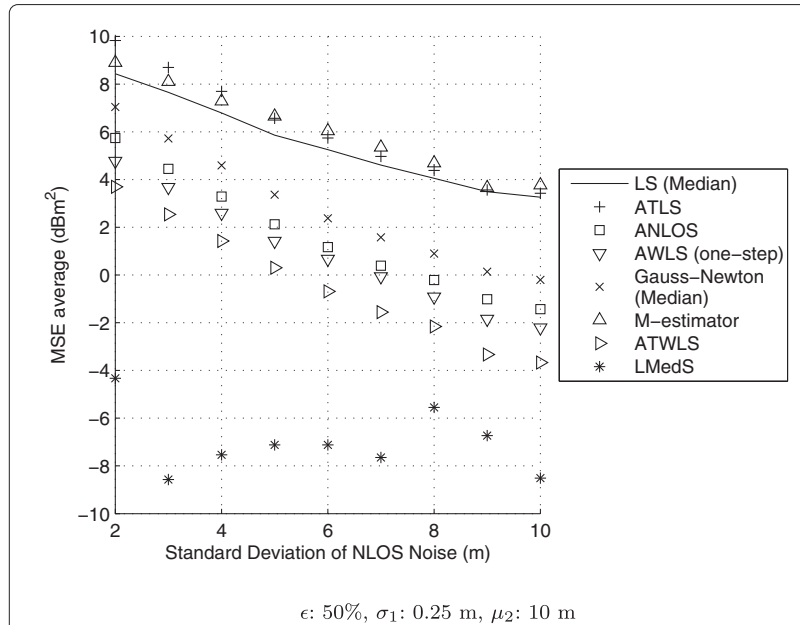

Fig. 8 Comparison of MSE averages of the proposed estimators with that of existing methods when sensors 3, 6 and 7 are LOS/NLOS sensors and the remaining sensors are LOS sensors $\left(\epsilon: 20 \%, \sigma_{1}: 0.25 \mathrm{~m}, \mu_{2}: 1 \mathrm{~m}\right)$

for the ATWLS algorithms performs the role of reducing the MSE average of the ATLS method and the MSE average performances of the proposed methods are nearly the same as can be seen from Fig. 2. Figures 3 and 4 assume the same condition as that in Fig. 2, with the exception that sensors 3 and 7 are the LOS/NLOS sensors in Fig. 3 and sensors 3, 6, and 7 are the LOS/NLOS sensors in Fig. 4. Again, the proposed adaptive methods using the WSE loss function outperformed the other existing methods from the results shown in Figs. 3 and 4. Figures 5, 6, 7, and 8 show the variation of the MSE average when the NLOS bias and contamination ratio is varied. The MSE average was larger as the contamination ratio and NLOS bias increased. The localization performance of the ATWLS

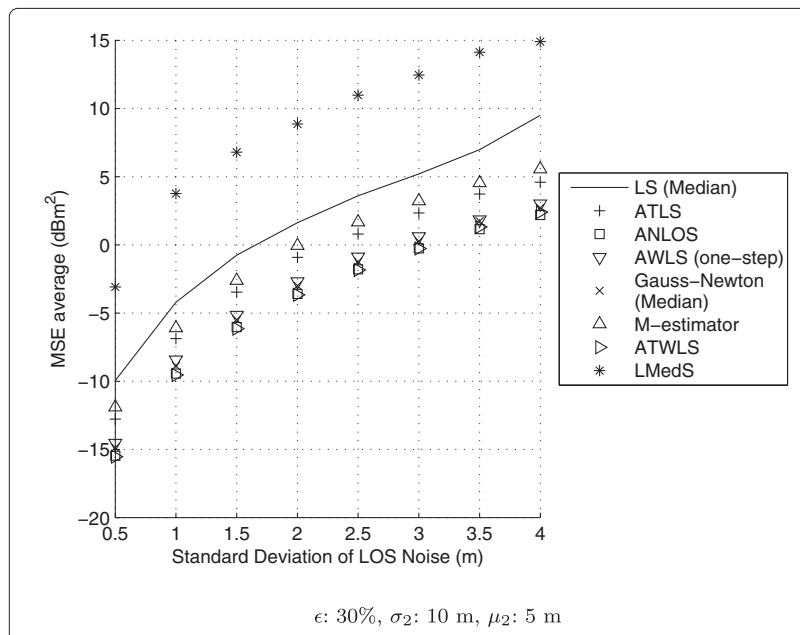

Fig. 9 Comparison of MSE averages of the proposed estimators with that of existing methods as a function of the standard deviation of LOS noise $\left(\sigma_{1}\right)$

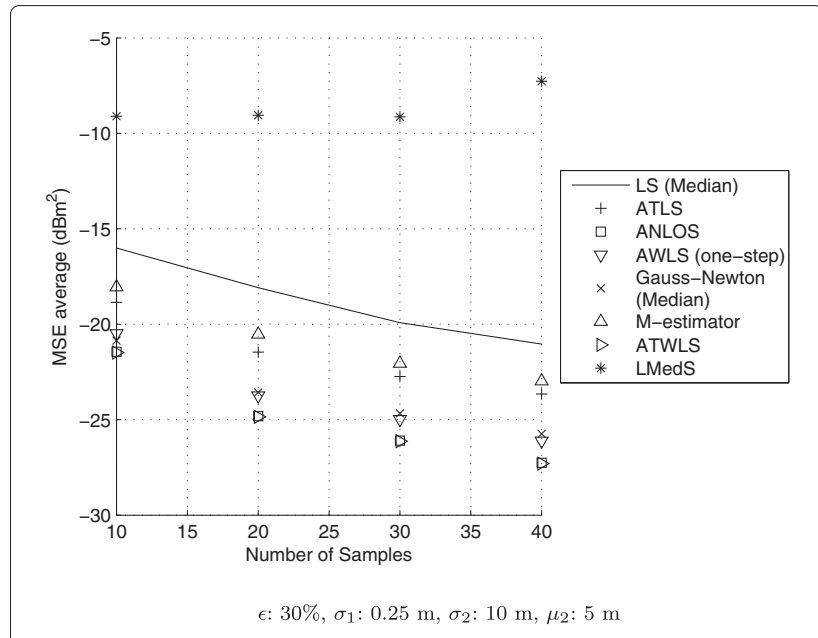

Fig. 10 Comparison of MSE averages of the proposed estimators with that of existing methods as a function of the number of samples

method was superior to the existing methods excluding Fig. 8. In particular, when the contamination ratio was $50 \%$, the MSE average drastically increased because the contamination ratio amounts to the breakdown point of median (50\%). Figure 9 shows the MSE average as a function of the standard deviation of LOS noise $\left(\sigma_{1}\right)$. The MSE average increased as the standard deviation of LOS noise increased and the ATWLS method outperformed the other existing algorithms. Figure 10 shows the MSE average as a function of the number of samples. The MSE average decreased as the number of samples increased excluding the LMedS method and the proposed ATWLS method was superior to the other existing algorithms. Figure 11 shows the MSE average of the proposed algorithms as a function of the number of sensors. In this case, the number of sensors increases from 5 to 9 and the number of LOS/NLOS sensors is fixed to three and the number

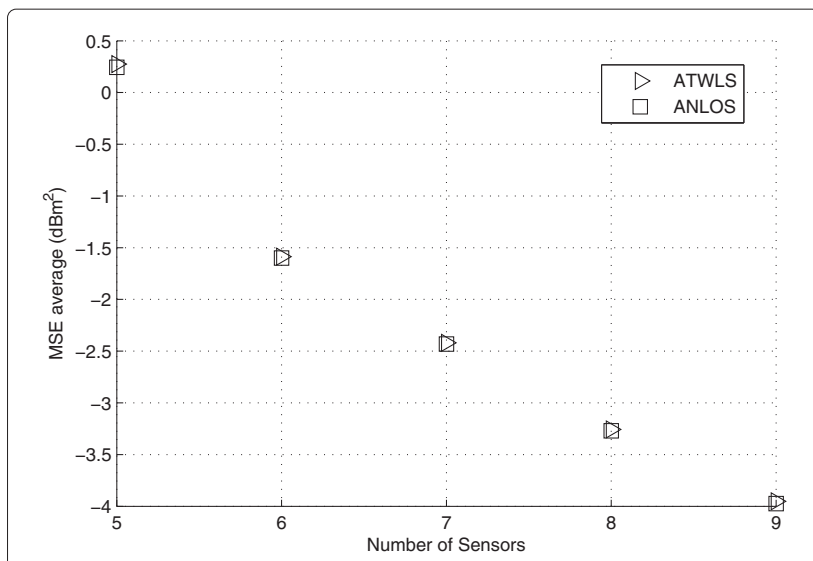

Fig. 11 Comparison of MSE averages of the proposed estimators as a function of the number of sensors (when the number of LOS sensors increases) 


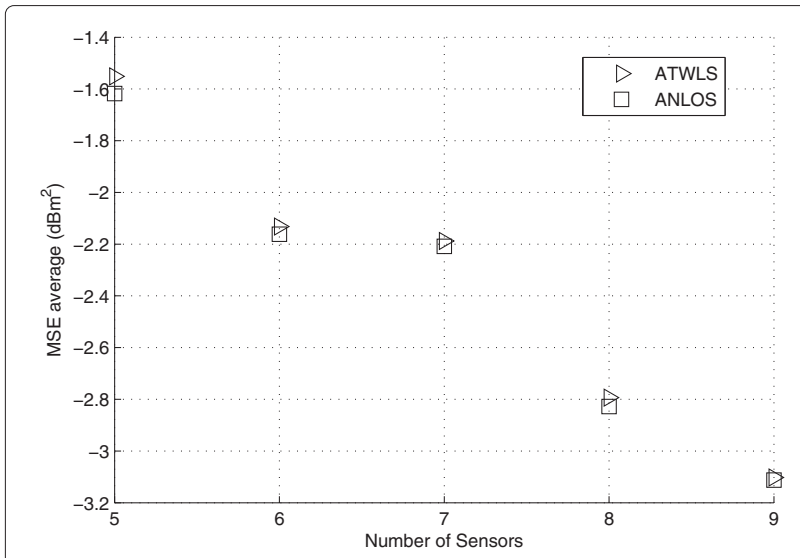

Fig. 12 Comparison of MSE averages of the proposed estimators as a function of the number of sensors (when the number of LOS/NLOS sensors increases)

of LOS sensors is increased. The standard deviation of the LOS noise was $0.75 \mathrm{~m}$, that of the NLOS noise was $10 \mathrm{~m}$, the bias was $1 \mathrm{~m}$, and the contamination ratio was $30 \%$. We can see that the MSE averages of the localization decrease as the number of sensors increases. Meanwhile, Fig. 12 shows the MSE averages of the proposed methods as a function of the number of sensors when the number of the LOS/NLOS sensors increases. The number of the LOS/NLOS sensors is one when the number of sensors is five and then increases in parallel with the number of sensors. The MSE averages of the proposed methods decrease as the number of LOS/NLOS sensors increases and the decreasing rate of the MSE averages is lower compared to the case in which the number of LOS sensors increases. Figure 13 shows the MSE averages as a function of the radius of the sensor network and the MSE averages decrease as the radius of the sensor network increases. The results of Figs. 11, 12, and 13 are consistent with that

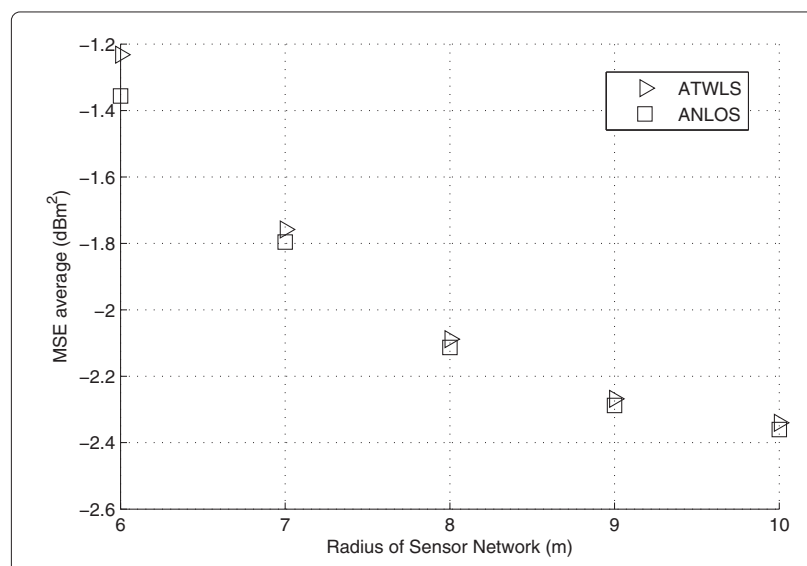

Fig. 13 Comparison of MSE averages of the proposed estimators as a function of the radius of sensor network

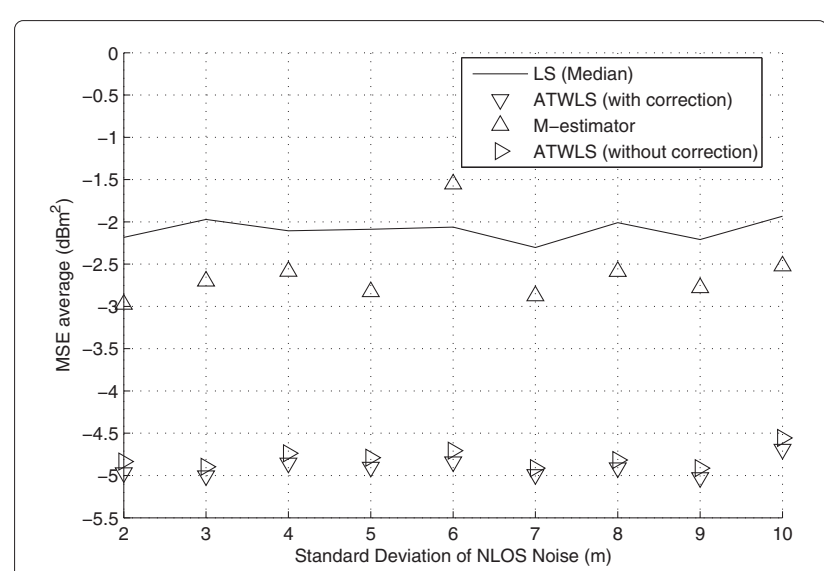

Fig. 14 Comparison of MSE averages of the proposed estimators with that of existing methods when the sensor position error exists

of [29]; that is, as the number of sensors are larger, the geometric dilution of precision (GDOP) is lower, on the other hand, as the number of sensors is smaller, the GDOP gets higher. The GDOP metric has been used as the indicator of the localization accuracy for given deployment of GPS systems and the localization accuracy is higher as the GDOP is lower. Also, the GDOP is low when the sensors are far apart; on the contrary, the GDOP is high when the sensors are close together. Figure 14 shows the MSE averages in the presence of the sensor position error. The variance of the sensor position error was $0.5 \mathrm{~m}^{2}$, the standard deviation of the LOS noise was $0.25 \mathrm{~m}$, the bias was $1 \mathrm{~m}$, the contamination ratio was $30 \%$, and sensors 3,6 , and 7 were the LOS/NLOS sensors. The localization performance of the ATWLS algorithm with the correction for the sensor position error described in Section 4.3 is superior to the ATWLS method without the correction for the sensor position error. Furthermore, the averaged computational time of the robust localization methods is shown in Table 2 to testify whether the proposed closedform ATWLS algorithm is more efficient than the other algorithms with respect to the computational complexity.

The computational time was averaged for 200 iterations. We can see that the averaged computational time of the ATWLS algorithm is lower than that of the other robust localization methods.

Table 2 Comparison of the computational time

\begin{tabular}{ll}
\hline Algorithm & Computational time (seconds) \\
\hline ATWLS & $3.0 \times 10^{-4}$ \\
ANLOS & $2.1 \times 10^{-3}$ \\
Gauss-Newton (median) & $1.1 \times 10^{-3}$ \\
M-estimator [16] & $1.1 \times 10^{-3}$ \\
LMedS [28] & $2.5 \times 10^{-3}$ \\
\hline
\end{tabular}




\section{Conclusions}

Robust LOS/NLOS mixture TOA source localization methods for multiple measurements using the error covariance of the sample mean and sample median were proposed. The proposed adaptive methods utilized the loss function as the WSE in which the sample mean was used when the sensor was identified as the LOS sensor, while the WSE loss function based on the sample median was employed when the testing result that the sensor is in the LOS/NLOS state is valid. The proposed estimators showed superior MSE average performances compared to those of the other localization methods using only the sample median, ATLS, and AWLS (one-step) estimators when the contamination ratio was moderate. In particular, the closed-form ATWLS method had similar MSE average performance to that of the proposed iteration-based method, preserving the advantages of low computational complexity and avoidance of the divergence problem of the solution. In addition, the proposed method was superior to the $M$-estimator and LMedS estimator.

\section{Acknowledgements}

This research was supported by Basic Science Research Program through the National Research Foundation of Korea (NRF) funded by the Ministry of Science, ICT and Future Planning (NRF-2014R1A2A1A10049735). This research was supported by the MSIP (Ministry of Science, ICT and Future Planning), Korea, under the ITRC (Information Technology Research Center) support program (IITP-2016-H8501-16-1016) supervised by the IITP (Institute for Information and communications Technology Promotion).

\section{Competing interests}

The authors declare that they have no competing interests.

\section{Author details}

${ }^{1}$ Department of Electronics and Computer Engineering, Hanyang University, Seoul 133-791, Republic of Korea. ${ }^{2}$ Department of Electronic Engineering, Hanyang University, Seoul 133-791, Republic of Korea.

Received: 4 January 2016 Accepted: 29 July 2016

Published online: 17 August 2016

\section{References}

1. DJ Torrieri, Statistical theory of passive location systems. IEEE Trans. Aerosp. Electron. Syst. 20(2), 183-198 (1983)

2. $\mathrm{YT}$ Chan, $\mathrm{KC} \mathrm{Ho}$, A simple and efficient estimator for hyperbolic location. IEEE Trans. Signal Process. 42(8), 1905-1915 (1994)

3. HC So, L Lin, Linear least squares approach for accurate received signal strength based source localization. IEEE Trans. Signal Process. 59(8), 4035-4040 (2011)

4. C Park, J-H Chang, Shrinkage estimation-based source localization with minimum mean squared error criterion and minimum bias criterion. Digital Signal Process. 29, 100-106 (2014)

5. JA Belloch, A Gonzalez, AM Vidal, M Cobos, On the performance of multi-GPU-based expert systems for acoustic localization involving massive microphone arrays. Expert Syst. Appl. 42, 5607-5620 (2015)

6. A Chatterjee, F Matsunog, A Geese PSO tuned fuzzy supervisor for EKF based solutions of simultaneous localization and mapping (SLAM) problems in mobile robots. Expert Syst. Appl. 37, 5542-5548 (2010)

7. I Guvenc, C Chong, A survey on TOA based wireless localization and NLOS mitigation techniques. IEEE Commun. Surv. Tutor. 11(3), 107-124 (2009)

8. AM Zoubir, V Koivunen, Y Chakhchoukh, M Muma, Robust estimation in signal processing: a tutorial-style treatment of fundamental concepts. IEEE Signal Process. Mag. 29(4), 61-80 (2012)
9. S Zhang, S Gao, G Wang, Robust NLOS error mitigation method for TOA-based localization via second-order cone relaxation. IEEE Commun. Lett. 19(12), 2210-2213 (2015)

10. G Wang, H Chen, Y Li, N Ansari, NLOS error mitigation for TOA-based Iocalization via convex relaxation. IEEE Trans. Wireless Commun. 13(8), 4119-4131 (2014)

11. RM Vaghefi, J Schloemann, RM Buehrer, in Proc. Positioning Navigation and Communication. NLOS mitigation in TOA-based localization using semidefinite programming (WPNC, Dresden, 2013), pp. 1-6

12. RM Vaghefi, RM Buehrer, Cooperative localization in NLOS environments using semidefinite programming. IEEE Commun. Lett. 19(8), 1382-1385 (2015)

13. PJ Rousseeuw, AM Leroy, Robust Regression and Outlier Detection. (Wiley, Hoboken, 1987)

14. Z Li, W Trappe, Y Zhang, B Nath, in Proc. IEEE Int. Symp. Information Processing in Sensor Networks. Robust statistical methods for securing wireless localization in sensor networks (IPSN, Los Angeles, 2005), pp. 91-98

15. PHuber, Robust Statistics, (Hoboken, 1981)

16. X-W Chang, Y Guo, Huber's M-estimation in relative GPS positioning: computational aspects. J. Geodesy. 79(6-7), 351-362 (2005)

17. JL Hodges, EL Lehmann, Estimates of location based on rank tests. Ann. Math. Stat. 34, 598-611 (1963)

18. FR Hampel, EM Ronchetti, PJ Rousseeuw, WA Stahel, Robust statistics: The approach based on influence functions. (Wiley, New York, 1986)

19. J Schroeder, S Galler, K Kyamakya, K Jobmann, in Proc. 4th Workshop on Positioning, Navigation and Communication. NLOS detection algorithms for ultra-wideband localization (WPNC, Hannover, 2007)

20. S Kay, Fundamentals of statistical signal processing, Vol. II: Detection Theory. (Prentice Hall, Upper Saddle River, 1998)

21. F Gustafsson, F Gunnarsson, Mobile positioning using wireless networks. IEEE Signal Process. Mag. 22, 41-53 (2005)

22. U Hammes, E Wolsztynski, AM Zoubir, Robust tracking and geolocation for wireless networks in NLOS environments. IEEE J. Sel. Top. Signal Process. 3(5), 889-901 (2009)

23. Y Feng, C Fritsche, F Gustafsson, AM Zoubir, TOA-based robust wireless geolocation and Cramer-Rao lower bound analysis in harsh LOS/NLOS environments. IEEE Trans. Signal Process. 61(9), 2243-2255 (2013)

24. S Bartoletti, W Dai, A Conti, MZ Win, A mathematical model for wideband ranging. IEEE J. Sel. Topics Signal Process. 9(2), 216-228 (2015)

25. P Rider, Variance of the median of small samples from several special populations. J. Am. Stat. Assoc. 55(289), 148-150 (1960)

26. M Sun, L Yang, KC Ho, Accurate sequential self-localization of sensor nodes in closed-form. Signal Process. 92(12), 2940-2951 (2012)

27. KC Ho, X Lu, L Kovavisaruch, Source localization using TDOA and FDOA measurements in the presence of receiver location errors: analysis and solution. IEEE Trans. Signal Process. 55(2), 684-696 (2007)

28. R Casas, A Marco, JJ Guerrero, J Falco, Robust estimator for non-line-of-sight error mitigation in indoor localization. Eurasip J. Adv. Signal Process. 2006(43429), 1-8 (2006)

29. H Godrich, AM Haimovich, RS Blum, Target localization accuracy gain in MIMO radar based system. IEEE Trans. Inf. Theory. 56(6), 2783-2803 (2010) 